\title{
Mortality Tempo: A Guide for the Skeptic ${ }^{*}$
}

\section{Griffith Feeney}

\begin{abstract}
The idea of mortality tempo effects derives from the idea of fertility tempo effects, which were introduced by Norman B. Ryder and are widely known and accepted. Given the essential similarity of the ideas, it might be regarded as curious that mortality tempo effects have only recently been recognized and are not as yet generally accepted. The explanation for this may be that mortality tempo effects have implications that seem to be at variance with established ways of modeling and analyzing mortality and population dynamics. This paper develops a discrete approach to describing and analyzing mortality tempo effects. The discrete approach is mathematically undemanding, yet powerful. It is used here, for example, to define age-specific tempo effects. The focus of this work is the measurement of length of life in empirical populations. It is shown that any empirical population may be approximated by a suitably constructed discrete model population.
\end{abstract}

Keywords: Mortality $\cdot$ Period mortality $\cdot$ Death rate $\cdot$ Tempo effects $\cdot$ Tempo distortion $\cdot$ Tempo adjustment $\cdot$ Mortality tempo $\cdot$ Bongaarts - Feeney

\section{Introduction}

Mortality tempo effects are proposed in several papers (Bongaarts/Feeney 2002, 2003, 2006; Bongaarts 2005; Feeney 2006). The idea derives directly from the idea of fertility tempo effects, which were introduced by Norman B. Ryder $(1956,1964)$ half a century ago and are widely known and accepted. For recent work and references see Bongaarts and Feeney (1998) and Zeng Yi and Land (2002).

Given the essential similarity of the ideas, it might be regarded as curious that mortality tempo effects have only recently been recognized and are not as yet (this based on discussions with colleagues) generally accepted (see Wachter 2005, Goldstein 2006, Guillot 2006). Only few publications used tempo-adjusted figures for

The paper is a revised version of a working paper with the same title which was published at www.gfeeney.com in 2003. 
analyzing international differences in life expectancy (Luy 2006; Luy/Wegner 2009). The explanation for this may be that mortality tempo effects have implications that seem to be at variance with established ways of modeling and analyzing mortality and population dynamics (see also Vaupe/ 2002, 2005).

This paper develops a discrete approach to describing and analyzing mortality tempo effects. The continuous mathematics of Bongaarts and Feeney $(1998,2002$, 2003,2006 ) tends to obscure what are at heart quite simple ideas. The discrete approach is mathematically undemanding, yet powerful. It is used here, for example, to define age-specific tempo effects, something that has yet to be done in the continuous formulation (a similar example can be found in Horiuchi 2005).

The focus of this work is the measurement of length of life in empirical populations, but it is convenient to begin by examining two simple model populations. The relevance of the models to empirical populations is established in paragraph 5 , in which it is shown that any empirical population may be approximated by a suitably constructed model population.

\section{The Simplest Case}

In the following, the idea of mortality tempo effects is illustrated by means of Lexis diagrams. The Lexis diagram is a useful device to clarify relations between exposure segments for cohorts and exposure segments for periods (Preston et al. 2001: $31 \mathrm{ff})$. In Figure 1a, the period, i.e. the calendar year, is displayed on the $\mathrm{x}$-axes for the years 1900 to 2000 . Age is displayed on the $y$-axes with age 0 at the top and age 90 at the bottom of the graph. The diagonal lines display the life lines of cohorts in which the demographic events occur. Let calendar years be divided into 0.2 year subintervals and consider a population closed to migration in which (1) all births occur at the midpoints of these intervals and (2) all deaths to any cohort occur at a single exact age. In figure 1a, age at death is constant at exact age 60 prior to 1925 , rises linearly at the rate of 0.2 years per year from 1925 through 1975, and is constant at exact age 70 from 1975 forward. Because of the limited graphical resolution available, only select cohort lines are shown.

This Lexis diagram would be an incomplete representation of the population even if all cohort lines were shown because each cohort line may represent many persons, and possibly different numbers of persons in different cohorts. The number of persons in each cohort must be specified exogenously. Mortality tempo results may be derived without any assumption about the numbers of persons in different cohorts, but simplifications result if every cohort contains the same number of persons.

Figure $1 \mathrm{~b}$ shows detail in the small rectangle in the vicinity of age 60 and 1925 in figure 1a. Cohort lines are shown in full detail, at 0.2 year intervals. Suppose that each life line represents 20,000 persons, for a total of 100,000 births annually. Then 100,000 deaths occur during 1924, when length of life is constant, but only 80,000 deaths occur during 1925 and 1926, during which life times are increasing. This decline in the annual number of deaths results from the increase in length of life. 
Fig. 1a: Lexis Diagram of a Simple Model Population

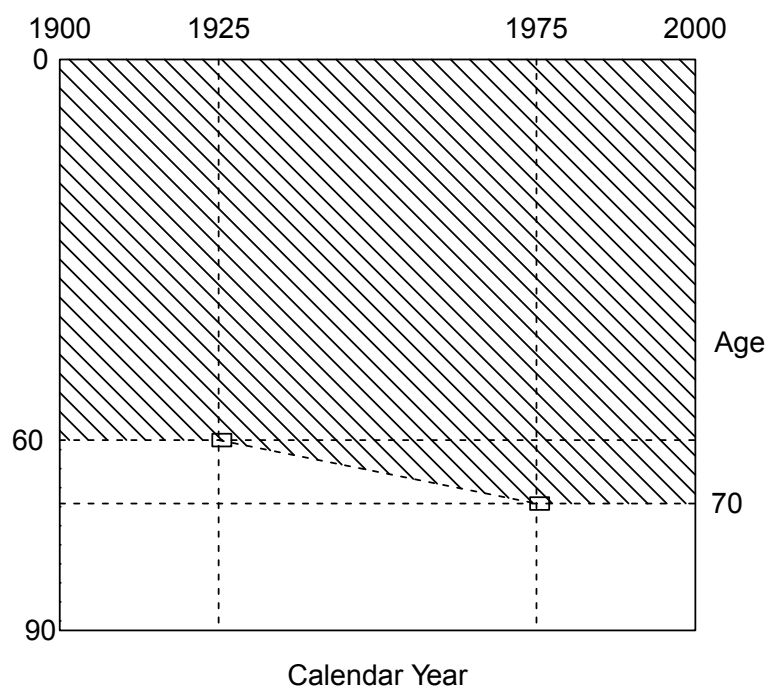

Source: own design

Fig. 1b: Lexis Diagram Detail circa 1925 and Age 60

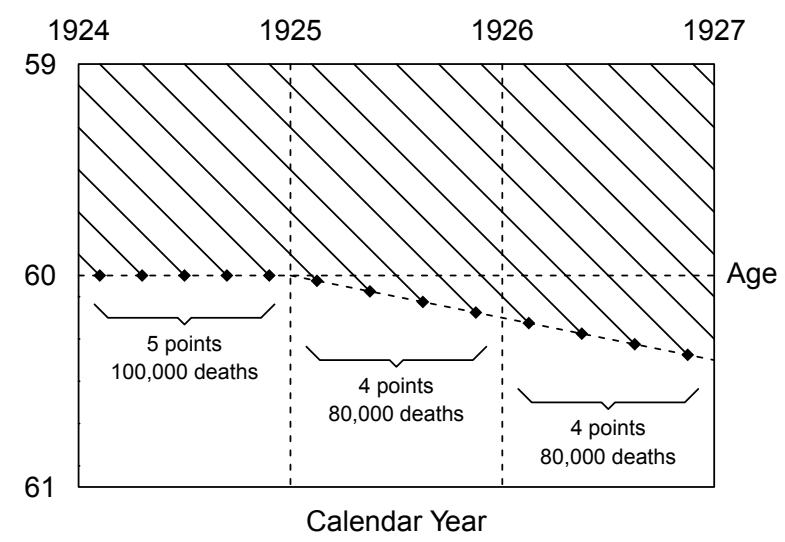

Source: own design

Since each cohort lives somewhat longer than the preceding cohort, the intervals between deaths are longer than the intervals between births. The deaths to the five cohorts that reach age 60 during 1925 are spread out over a period longer than one year. The deaths of persons in the last of the five cohorts shown are "bumped" to 1926. 
This decline in the annual number of deaths is transitory in the sense that it will disappear when (if) length of life stops rising (it is conceivable that length of life will rise indefinitely). This is illustrated in figure 1c, which shows detail in the small rectangle in the vicinity of age 70 and 1975 in figure 1a.

During 1974, length of life is still rising. Intervals between deaths are therefore still longer than intervals between births, and only 80,000 deaths occur during this year. In 1975 and 1976, however, length of life is no longer rising and the number of deaths rises back to 100,000 per year.

Unequal numbers of persons in different birth cohorts would change this example in detail without affecting the essential message. If annual numbers of births were increasing exponentially, for example, as they would in a stable population, numbers of deaths before 1925 and after 1975 would change continuously and the number of births in the last quarter of the century would be much larger than the number in the first quarter of the century, but the number of deaths would decline sharply between 1924 and 1925 and rise sharply between 1974 and 1975.

Consider now the trend of the crude death rate in this population. Prior to 1925, $6,000,000$ person years are lived by the population each year: 100,000 person years by persons aged $0,1, \ldots, 59$. Similarly, $7,000,000$ person years are lived by the population in 1975 and each later year.

The annual number of deaths to the population is the same before and after the rise in length of life: 100,000. The net decline in the crude death rate, from 16.7 per thousand to 14.3 per thousand, is due entirely to the increase in its denominator.

The situation is different during the 50 year period when age at death is rising. During this period annual person years lived by the population increases linearly at the rate of 20,000 person years per year from 6,010,000 in 1925 to 6,990,000 in 1974. The annual number of deaths is constant at 80,000 per year. The crude death rate falls from 13.3 per thousand in 1925 to 11.4 per thousand in 1974.

Fig. 1c: Lexis Diagram Detail circa 1975 and Age 70

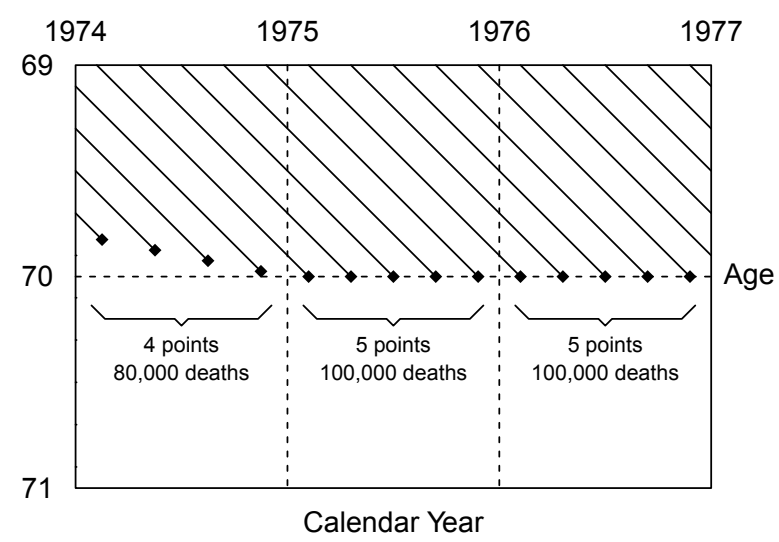

Source: own design 
These changes are summarized in figure 2 below. The net change in the crude death rate is modest (a $14 \%$ decline) and plausible in relation to the magnitude of the change in length of life (a $17 \%$ increase). The rate does not move smoothly from the higher to the lower level, however, as indicted by the sloping dotted line. Rather it falls abruptly to a level well below the eventual level at the beginning of the period of increase, only to rise abruptly to this eventual level at the end of the period of increase.

The net change reflects the increase in the denominator of the rate, which is a direct result of longer length of life. The pattern of change - the sudden drop and subsequent recovery - reflects mainly the decrease in the numerator of the rate from 100,000 to 80,000 deaths and the subsequent rise back to 100,000 deaths. During the transition, the rise in the denominator plays a minor role.

Note that the crude death rate does not decline linearly during 1925-1975, though no curvilinearity is visible in the plot, since the denominator of the rate is constant and the enumerator increases linearly.

Fig. 2: Trend in the Crude Death Rate with Increasing Length of Life

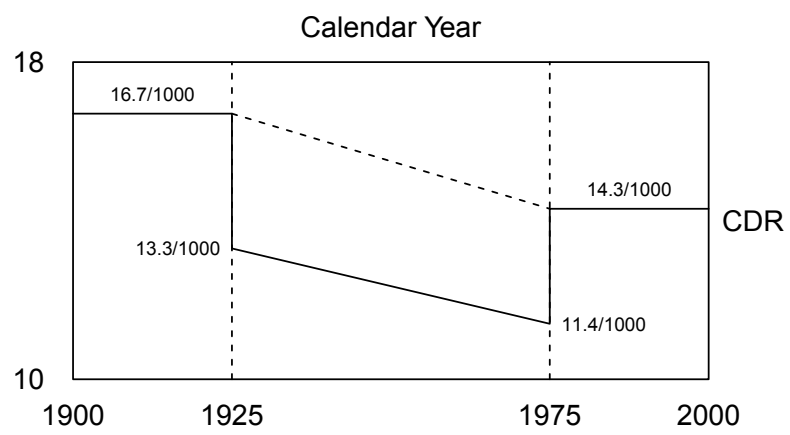

Source: own design

\section{$3 \quad$ Relationships}

When cohort size is constant (constant annual births), there is a simple relationship between the rate of change in age at death and the magnitude of the tempo effect. To see what the relationship is consider figure $3 a$, which elaborates figure $1 \mathrm{~b}$. At the rate of increase in age at death illustrated here, 0.2 years per year, deaths of persons reaching exact age 60 during the last 0.2 year subinterval of the year (note the tick marks indicating the division of the year into 0.2 year intervals) will be bumped to 1926.

The equality of these magnitudes is not coincidental. Observe the small, shaded right triangle. The length of the right side of this triangle, 0.2 years, equals the annual rate of increase in length of life. The length of its top side represents the persons whose death will be bumped into 1926 . 
Fig. 3a: Lexis Diagram Detail Illustrating "Bumping" (0.2 year intervals)

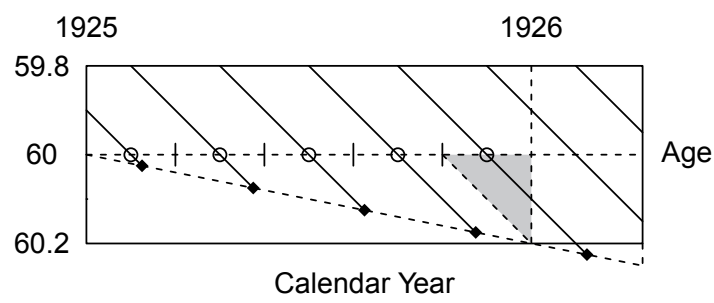

Source: own design

If each cohort line in the figure represents the same number of persons, the distribution of times at which people arrive at exact age 60 is (to within the limits imposed by the length of the interval between births) uniform. The length of the top side of the shaded triangle will then give the proportion of these persons whose death will be "bumped" from 1925 to 1926 by the increase in length of life. If $r$ denotes the annual rate of change in age at death, then, the tempo effect reduces the annual number of deaths by a factor of $1-r$.

Of course the finite interval between births results in some "stickiness" in the effects. With a 0.2 year interval between cohorts, for example, a slightly more or less rapid rate of increase in length of life would not have any effect on the number of deaths bumped to the next year. But the choice of the 0.2 year interval is inessential to the logic of the argument. It simply makes for a clear diagram.

In figure $3 \mathrm{~b}$, for example, the interval between cohorts is halved to 0.1 year, so that the annual number of cohorts is doubled to 10 . In figure $3 c$ the interval between cohorts is halved again to 0.05 years, and the annual number of cohorts doubled correspondingly to 20 . The tempo effect is $1-r$ in each case, and by the same argument given for figure $3 \mathrm{a}$.

The uniform births (constant cohort size) assumption is necessary for the $1-r$ relation to hold, but tempo effects are general. To calculate their magnitude it is only necessary to know how many persons reached exact age 60 during the last fifth of

Fig. 3b: Lexis Diagram Detail Illustrating "Bumping" (0.1 year intervals)

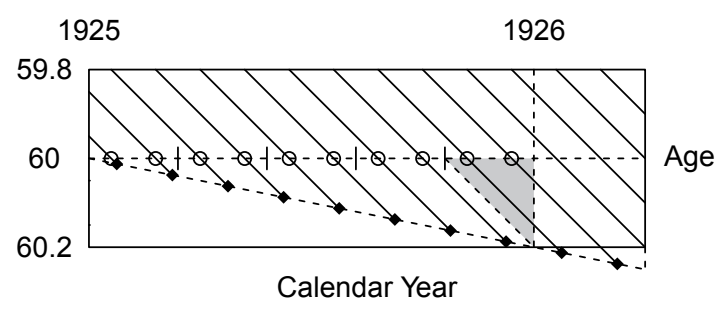

Source: own design 
Fig. 3c: Lexis Diagram Detail Illustrating "Bumping" (0.05 year intervals)

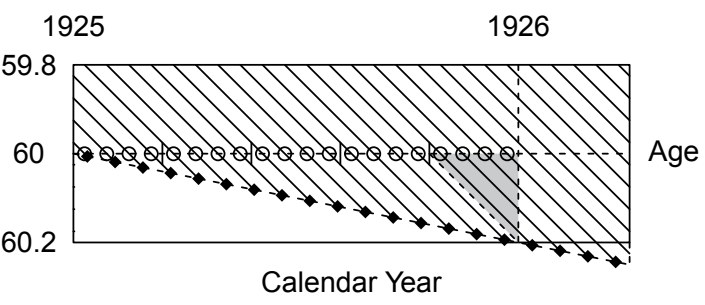

Source: own design

the year. This determines the number of deaths that will be bumped to the following year.

Nor does the logic of the argument depend on what happens before or after a period during which length of life increases. Observing only the data shown in figures 3a-c, it is evident that (1) length of life was increasing during this period by 0.2 years per year and that (2) more deaths would have occurred during the year $(20,000$ more, given the uniform births assumption) if this increase in length of life had not occurred. This is a simple consequence of what Norman B. Ryder once called "the most important fact in demography: we all get one year older every year. ${ }^{\prime 1} \mathrm{It}$ is impossible to defer age at death without also deferring time of death.

\section{$4 \quad$ Age-Specificity}

It is well known that changes in age distribution during an idealized demographic transition result in a temporary decline in the crude death rate well below its long term levels (Feeney/Mason 2001: 63-64). In view of this, the pattern of change in crude death rates displayed in Figure 2 may be regarded as unsurprising. In this section, however, we show that the same effect occurs for age-specific death rates.

Consider a population in which all births occur in bursts at 0.2 year intervals, as before, but in which all deaths occurring during some base year occur at exactly the midpoint of a single year of age between 0 and 99 years. Suppose that, at the end of this base year, age at death within each age group begins to increase linearly at the rate of 0.2 years per year for all persons, and ceases increasing at the end of the year, resulting in the pattern of life lines shown in figure 4.

Assume further, for the moment, that annual numbers of births to the population have been constant, at least from time $(t-1)-(x+2)$ to time $(t+2)-(x-1)$, and that the proportion of deaths in any birth cohort that occur at the $i$-th age at death is constant over all cohorts. These two assumptions imply that each "dot" in figure 4 represents the same number of deaths.

1 Oral statement of Norman B. Ryder. 
Let $N_{x}$ denote the number of persons reaching exact age $x$ during each of the three years shown, and let $D_{x}$ denote the number of deaths during the first and third years. The number of deaths during the middle year is $20 \%$ less than $D_{x}$. Person years lived at age $x$ in completed years in the first through the third years are $N_{x}-0.5 D_{x^{\prime}} N_{x}-0.4 D_{x^{\prime}}$ and $N_{x}-0.3 D_{x^{\prime}}$, respectively, whence the age-specific death rates for the three years are

$$
\frac{D_{x}}{N_{x}-0.5 D_{x}}, \frac{0.8 D_{x}}{N_{x}-0.4 D_{x}} \text {, and } \frac{D_{x}}{N_{x}-0.3 D_{x}}
$$

The subtracted terms in the denominators represent person years not lived by the persons who die at age $x$ in completed years. Because of the rise in age at death, this term declines each year.

The quantities (1) may be expressed as

$$
\frac{1}{1 / q_{x}-0.5}, \frac{0.8}{1 / q_{x}-0.4} \text {, and } \frac{1}{1 / q_{x}-0.3} \text {, }
$$

where $q_{x}$ denotes $D_{x} / N_{x^{\prime}}$ the proportion of persons reaching exact age $x$ who die before reaching exactly age $x+1$. With high life expectancy at birth, values for young and middle adult ages will be on the order of 0.01 or 0.001 , the value of $1 / q_{x}$ will be 100 to 1,000 , and the impact of the subtractions in the denominator will be negligible.

This shows that, for the hypothesized model population, age-specific death rates for all but the oldest ages will decline by approximately $20 \%$ between year $t-1$ and year $t$ and rise by approximately $25 \%$ between year $t$ and year $t+1$. The result is approximate rather than exact only because of the slight differences in the denominators. This temporary decline in age-specific death rates is a tempo effect. It results from a decline in the numerators of the rates rather than from an increase in their denominators.

Figure 4 shows length of life increasing at the same rate in each of the three age groups shown, but this is inessential to the logic of the argument. If the ages of death in the three age groups increase at different rates, different numbers of deaths will be "bumped" and the magnitude of the tempo effect will vary from one age group to another. Given the requisite information, however, these rates and the corresponding numbers of bumped deaths may be calculated exactly for each age group. In particular, with uniform births, the number of deaths at age $x$ will be deflated by the factor $1-r_{x}$ where $r_{x}$ denotes the rate of increase in age at death for age $x$.

We see, then, that tempo effects operate for age-specific death rates exactly as they do for the crude death rate in the simple case in which all deaths to every cohort occur at a single exact age. Given sufficiently detailed data, tempo adjustments may be made age-specifically. The assumption of a constant rate of increase in length of life in adult ages made in Bongaarts and Feeney $(2002,2003)$ is a good 
Fig. 4: $\quad$ Lexis Diagram Detail Illustrating Deaths at Multiple Ages

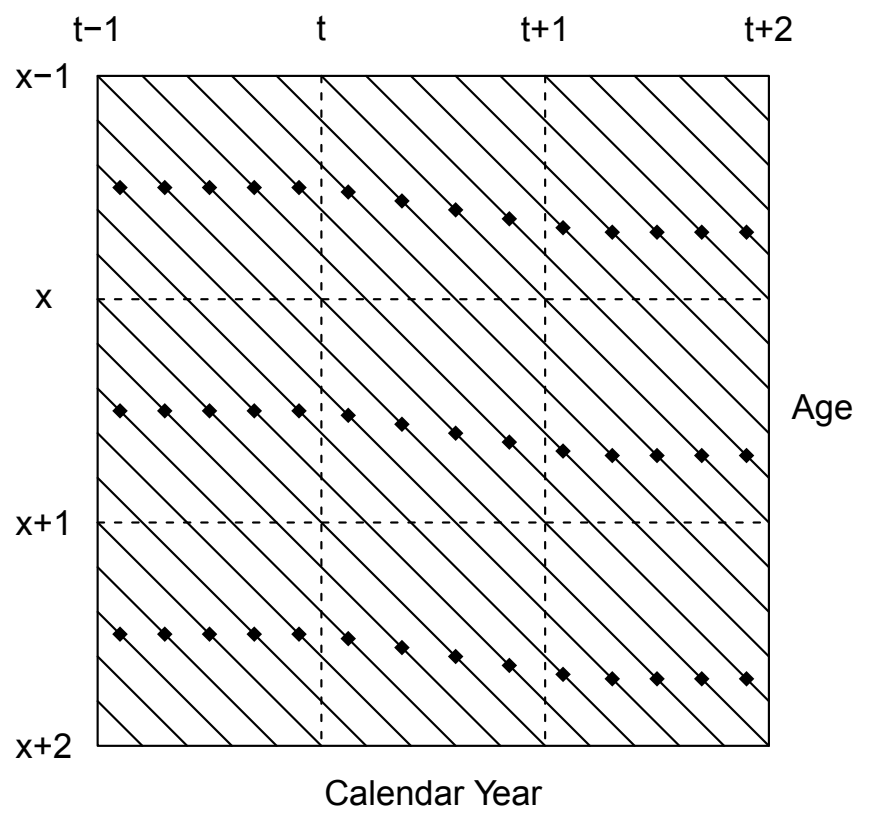

Source: own design

approximation for high life expectancy populations, but tempo effects are not restricted to or in any way dependent on this assumption. They may be observed in any population and at any level of life expectancy at birth.

\section{$5 \quad$ Approximating Empirical Populations}

The model populations considered above are obviously artificial, and have been constructed specifically to reveal the operation of mortality tempo effects. Is it valid to infer, on the basis of these models, that tempo effects occur in empirical populations? It is shown in this section that any empirical population may be approximated by a model population having all the essential characteristics of the model populations discussed above. Tempo effects may not be visible in empirical populations as they are in figure 4, but they occur in the same way, and may be revealed in the same way, by constructing an approximating model population. The model construction is as follows.

(1) "Adjust" the time of birth of every member of the population to the nearest multiple of $\Delta$ years, $\Delta>0$, with any births occurring exactly half way between two such times adjusted to the following multiple.

(2) Select an initial birth cohort and an initial set of age intervals and compute the proportions of deaths that occur in each age interval for this initial cohort. 
(3) Define age intervals for each following cohort in such a way that the proportion of deaths occurring in each age interval for the cohort equals the proportion of deaths in the corresponding age interval in the first cohort.

(4) "Adjust" the age at death for every person in every cohort to the average age at death for persons dying in the same "customized" age interval in this cohort.

These four steps define a model population that approximates the empirical population, but for which (1) all births occur in bursts at $\Delta$ year intervals, (2) all deaths to members of any cohort occur at one of a fixed number of exact ages (different ages, in general, for different cohorts), and (3) the proportion of total deaths that occur at the $i$-th age is the same for all cohorts. Note that the "customized" age groups of the preceding paragraph are a temporary "scaffolding" used to construct the model. They make no appearance in the final result.

Imagine now the Lexis diagram representation of this approximating model population in the manner of figure 4. If length of life in the empirical population does not change, the diagram will consist of cohort lines and dots representing deaths, like those in figure 4, but with all rows of dots falling on horizontal lines. If length of life increases, some or all of the rows of dots will turn down to higher ages at death. If length of life decreases, some or all of the rows of dots will turn up to lower ages. It is possible that, for a time, different rows of dots move in different directions. By construction, however, the rows of dots can never cross each other because i-th row, numbering from the top, corresponds to deaths occurring at the $i$-age age at death within each cohort.

A model population constructed in this way will present a more complex picture than figures 4 in three respects. First, cohorts sizes will not in general be the same, so the number of deaths represented by the points for the $i$-th age at death for different cohorts will not necessarily be the same. Second, the series of dots for the $i$-th age at death will in general move irregularly. Third, when calendar years and single years of age are marked off, the series of points will not necessarily remain within single years of age during calendar years.

The first difference complicates the derivation of general formulas, but tempo affects may be calculated directly, given the requisite data, by the method indicated above. The second difference may be addressed by making the series of dots piecewise linear. The third difference requires consideration of "bumping" to the next age group as well as to the next year.

These differences between empirical populations and the approximating model populations complicate the calculation of tempo effects, and require unconventionally detailed data, but they do not affect the essential conclusion that increasing (declining) length of life results in a temporary decline (increase) in age-specific death rates. 


\section{Discussion}

The single age at death example of paragraph 2 shows how tempo effects operate when they take the form of a steady increase in the interval between two events. (There are of course other kinds of tempo effects, due for example to temporary postponement of births due to social disruption or temporary increases in numbers of deaths due to war or epidemics; the analysis in this paper does not apply to tempo effects of this kind.) When length of life increases, intervals between deaths rise above corresponding intervals between births. The number of deaths occurring during any particular time period is therefore less than it would have been if the rise in length of life had not occurred. A modest increase in length of life $(0.2$ years per year) substantially decreases the number of deaths (by $20 \%$ ).

This establishes the existence of mortality tempo effects, but the age-specific example of paragraph 4 is required to demonstrate their importance. The age-specific example shows that, for a model population similar to empirical populations, mortality tempo operates in essentially the same way as in the single age at death example. The procedure of paragraph 5 for approximating empirical populations effectively extends this result to all populations.

It is worth revisiting figure 4 and formula (2) with some specific magnitudes. For United States females in the year 2000, $q_{x}$ values for ages $40,50,60,70$ and 80 were, respectively, 0.0015, 0.0032, 0.0080, .0200 and 0.0506 (Bell/Miller 2002; the tables are for the United States Social Security Area). For the first four of these ages, the change in the denominator between the first and the last year is all but negligible. With $q_{70}=0.02$, for example, the age-specific death rate for age 70 declines from 0.020202 in the first year to 0.020121 in the last year, a decline of $0.4 \%$. Even with $q_{80}=0.05$, the decline is only $1.0 \%$. In contrast, the change in the numerator - the tempo effect - is $20 \%$.

It is difficult escape the conclusion that age-specific death rates are a poor indicator of changing length of life. Length of life over the three year period rises by 0.2 years, for the age groups shown, and the difference between the age-specific rates in the first and last year, though variable and increasing with age, is only $1 \%$ even at age 80 years. During the middle year, however, the age-specific death rate is $20 \%$ lower than the rates in both the first and the third year. An assessment of length of life based on these rates will evidently be very wide of the mark (see empirical estimates of tempo-adjusted life expectancy in Luy/Wegner 2009). Hence, tempo effects might have significant impact on the analysis of mortality trends and differentials as demonstrated by Luy $(2006,2008)$. For the case of mortality differences between eastern and western Germany, he has shown that the picture drawn by tempo-adjusted life expectancy differs from that painted by conventional life expectancy. Moreover, he concluded that the tempo-adjusted figures better fit expected trends in changing mortality and self-reported health among eastern and western Germans than the conventional figures.

The conclusion, then, seems clear: when length of life is increasing, age-specific death rates are "too low", in the sense that they are much lower than they will be when (if) length of life stops increasing. And if length of life is decreasing, age- 
specific death rates are "too high", in the sense that they are much higher than they will be when length of life stops decreasing.

This conclusion has striking implications for population dynamics. In the classical view, a cessation of change in age-specific death rates signals that change in length of life has ceased. The result of fixed age-specific death rates from this point in time forward is a more or less extended period during which the population age distribution moves toward equilibrium with the age-specific mortality risks.

But if age-specific death rates are "too low" when length of life is increasing, it is illogical to stipulate that they will remain constant when length of life stops increasing. Tempo effects imply that age-specific death rates will change when length of life stops increasing, specifically, that they will rise.

Referring again to figure 4 above, consider what will happen in the future if there is no further change in length of life. The obvious answer is that the ages at death to the right of those shown will be the same as the rightmost point in the plot. The point here is not whether this will happen, but that this is the only thing we can reasonably understand by "no further change". But this implies that, for the uniform births case, at least, the population age distribution will cease to change as soon as the increase in lifetime stops.

\section{Conclusion}

In the classical view, mortality is defined by risks of death, captured empirically by age-specific death rates. The idea that these rates can be "too low" seems to undermine concepts and tools for the analysis of mortality that have been established for well over a century. This is an alarming prospect. Let us strive to assimilate it.

Tempo effects, long recognized and accepted in the study of fertility, appear to be equally pertinent to the study of mortality, and in fact, to the study of demographic events generally. If this is so, they are pertinent not only to demographic analysis generally, but to all disciplines that work with similar concepts, such as biostatistics, failure theory and multivariate hazards modeling in economics and sociology.

Age-specific death rates (and occurrence-exposure rates generally) embody the principle of exposure to risk, arguably the most fundamental principle of classical demographic measurement. The principle is simply that numbers of events must be assessed in relation to the numbers of persons who might have experienced these events and the length of time during which they might have experienced these events.

The mortality tempo argument suggests that numbers of events must also be assessed in relation to changes in the tempo of these events, and that this is conceptually as fundamental as the principle of exposure to risk. The principle of exposure to risk is recognized by dividing numbers of events by the appropriate "exposure". Exposure to risk deals with denominators. Changes in tempo change the number of events in the numerators of occurrence-exposure rates and must be controlled for by a suitable "tempo adjustment". Tempo effects work on numerators. 
In classical demographic analysis, length of life is a residual quantity: it is what is left when the various risks of death have played themselves out. This is reflected in the most fundamental terminology of the subject. We speak first of "mortality", not of length of life. (Arguably it should be the other way around.) Deaths and death rates come first. Length of life comes only later, on incorporating death rates into a life table.

The mathematics of this way of thinking was developed long ago and becomes second nature to anyone trained in demography. This way of thinking is nonetheless a model. It is not the actuality. The actuality consists simply of pairs of numbers giving time of birth and time of death for all persons in a suitably defined set of persons. The familiar model provides a very useful way to think about the actuality and to work with human mortality data. It is not the only model, however, as Euclidian geometry is the only geometry, and it may not be the only useful model.

Consideration of tempo effects suggests that it may be useful to model length of life more directly, to develop mathematical models in which "increments to life" rather than "risks of death" play the fundamental role (see Feeney 2006).

\section{References}

Bell, Felicitie C.; Miller, Michael 2002: Life Tables for the United States Social Security Area 1900-2100. In: Actuarial Study 116. Washington, DC: United States Social Security Administration. [http://www.ssa.gov/OACT/NOTES/actstud.html, 30.09.2003].

Bongaarts, John 2005: Five period measures of longevity. In: Demographic Research 13,21: 547-558 [doi: 10.4054/DemRes.2005.13.21].

Bongaarts, John; Feeney, Griffith 1998: On the quantum and tempo of fertility. In: Population and Development Review 24,2: 271-291 [doi: 10.2307/2807974].

Bongaarts, John; Feeney, Griffith 2002: How long do we live? In: Population and Development Review 28,1: 13-29 [doi: 16.1111/j.1728-4457.2002.00013.x].

Bongaarts, John; Feeney, Griffith 2003: Estimating mean lifetime. In: Proceedings of the National Academy of Science 100,23: 13127-13133 [doi: 10.1073/pnas.2035060100].

Bongaarts, John; Feeney, Griffith 2006: The quantum and tempo of life-cycle events. In: Vienna Yearbook of Population Research: 115-151 [doi: 10.1553/populationyearbook2006s115].

Feeney, Griffith 2006: Increments of life and mortality tempo. In: Demographic Research 14,2: 27-46 [doi: 10.4054/DemRes.2006.14.2].

Feeney, Griffith; Mason, Andrew 2001: Population in East Asia. In: Mason, Andrew (Eds.): Population Change and Economic Development in East Asia: Challenges Met, Opportunities Seized. Stanford, California: Stanford University Press: 61-95.

Goldstein, Joshua R. 2006: Found in translation? A cohort perspective on tempoadjusted life expectancy. In: Demographic Research 14,5: 71-84 [doi: 10.4054/ DemRes.2006.14.5].

Guillot, Miche/ 2006: Tempo effects in mortality: An appraisal. In: Demographic Research 14,1: 1-26 [doi: 10.4054/DemRes.2006.14.1].

Horiuchi, Shiro 2005: Tempo effect on age-specific death rates. In: Demographic Research 13,8: 189-200 [doi: 10.4054/DemRes.2005.13.8]. 
Luy, Marc 2006: Mortality tempo-adjustment: an empirical application. In: Demographic Research 15,21: 561-590 [doi: 10.4054/DemRes.2006.15.21].

Luy, Marc 2008: Mortality tempo-adjustment: theoretical considerations and an empirical application. In: Barbi, Elisabetta; Bongaarts, John; Vaupel, James W. (Eds.): How long do we live? Demographic models and reflections on tempo effects. Berlin et al.: Springer: 203-233.

Luy, Marc; Wegner, Christian 2009: Conventional versus tempo-adjusted life expectancy - which is the more appropriate measure for period mortality? In: Genus 65,2: 1-28.

Preston, Samuel H.; Heuveline, Patrick; Guillot, Michel 2001: Demography. Measuring and modeling population processes. Oxford: Blackwell.

Ryder, Norman B. 1956: Problems of trend determination during a transition in fertility. In: Milbank Memorial Fund Quarterly 34,1: 5-21.

Ryder, Norman B. 1964: The process of demographic translation. In: Demography 1,1: 74-82.

Vaupel, James W. 2002: Life expectancy at current rates vs. current conditions: A reflexion stimulated by Bongaarts and Feeney's 'How long do we live?'. In: Demographic Research 7,8: 365-377 [doi: 10.4054/DemRes.2002.7.8].

Vaupel, James W. 2005: Lifesaving, lifetimes and lifetables. In: Demographic Research 13,24: 597-614 [doi: 10.4054/DemRes.2005.13.24].

Wachter, Kenneth W. 2005: Tempo and its tribulations. In: Demographic Research 13,9: 201-222 [doi: 10.4054/DemRes.2005.13.9].

Zeng, Yi; Land, Kenneth C. 2002: Adjusting period tempo changes with an extension of Ryder's basic translation equation. In: Demography 39,2: 269-285 [doi: 10.1353/ dem.2002.0022]

A German translation of this reviewed and author's authorised original article by the Federal Institute for Population Research and Dr. Marc Luy is available under the title "Tempo-Effekte in der Mortalität: ein Wegweiser für Skeptiker", DOI 10.4232/10.CPoS-2010-12de or URN urn:nbn:de:bibcpos-2010-12de7, at http://www.comparativepopulationstudies.de. 


\section{Comparative Population Studies - Zeitschrift für Bevölkerungswissenschaft}

www.comparativepopulationstudies.de

ISSN: 1869-8980 (Print) - 1869-8999 (Internet)

Published by / Herausgegeben von

Prof. Dr. Norbert F. Schneider

Layout and print: Federal Institute for Population Research, Wiesbaden (Germany)

Managing Editor / Redaktion

Frank Swiaczny

\section{Copy Editor / Schlussredaktion}

Dr. Evelyn Grünheid

\section{Scientific Advisory Board / Wissenschaftlicher Beirat}

Jürgen Dorbritz (Wiesbaden)

Paul Gans (Mannheim)

Johannes Huinink (Bremen)

Marc Luy (Wien)

Clara H. Mulder (Groningen)

Notburga Ott (Bochum)

Peter Preisendörfer (Mainz)

\section{Board of Reviewers / Gutachterbeirat}

Martin Abraham (Erlangen)

Laura Bernardi (Lausanne)

Hansjörg Bucher (Bonn)

Claudia Diehl (Göttingen)

Andreas Diekmann (Zürich)

Gabriele Doblhammer-Reiter (Rostock)

Henriette Engelhardt-Wölfler (Bamberg)

E.-Jürgen Flöthmann (Bielefeld)

Alexia Fürnkranz-Prskawetz (Wien)

Beat Fux (Zürich)

Joshua Goldstein (Rostock)

Karsten Hank (Mannheim)

Sonja Haug (Regensburg)

Franz-Josef Kemper (Berlin)

Michaela Kreyenfeld (Rostock)

Aart C. Liefbroer (Den Haag)

Kurt Lüscher (Konstanz)

Dimiter Philipov (Wien)

Tomáš Sobotka (Wien)

Heike Trappe (Rostock) 